\title{
O NÃO COMPARECIMENTO AO EXAME PREVENTIVO DO CÂNCER DE COLO UTERINO: RAZÕES DECLARADAS E SENTIMENTOS ENVOLVIDOS*
}

\author{
Marcele Peretto1, Luciana Balestrin Redivo Drehmer², Heloísa Maria Reckziegel Bello ${ }^{3}$
}

\begin{abstract}
RESUMO: O câncer do colo uterino apresenta altas taxas de prevalência e mortalidade e a principal estratégia para seu rastreamento é o exame citopatológico. Este estudo teve por objetivo conhecer os motivos pelos quais ocorre o não comparecimento na coleta do referido exame em uma Unidade de Saúde da Família de Porto Alegre - Rio Grande do Sul. Trata-se de um estudo qualitativo, cujos dados foram coletados em abril de 2011 por meio de entrevista semiestruturada e questionário sociodemográfico, aplicados a oito sujeitos. Os dados foram analisados pela técnica de análise de conteúdo revelando multiplicidade de razões e sentimentos envolvidos na realização do exame citopatológico, principalmente oriundos do território da sexualidade e da finitude da vida. Considera-se indispensável o vínculo entre profissionais e usuárias para que os sentimentos possam ser minimizados e haja maior adesão ao processo preventivo dessa doença.

PALAVRAS-CHAVE: Esfregaço vaginal; Saúde da mulher; Neoplasias do colo do útero; Emoções.
\end{abstract}

\section{FAILURE TO ATTEND CERVICAL CANCER SCREENING TESTS: REASONS GIVEN AND FEELINGS INVOLVED}

\begin{abstract}
Cervical cancer has high rates of prevalency and mortality and the principal strategy for tracking it is the Papanicolaou test. The objective of this study was to learn about the reasons which are behind failure to attend Family Health Units in Porto Alegre (state of Rio Grande do Sul) for the collection phase of this exam. It is a qualitative study, whose data was collected in April 2011 via semi-structured interviews and a socio-demographic questionnaire, completed by eight persons. The data was analyzed by the technique of content analysis, revealing a plethora of reasons and feelings linked with taking the Papanicolaou test, principally to do with the area of sexuality and the finite nature of life. The link between health professionals and health service users is indispensable for such feelings to be minimized and compliance with measures for preventing this disease to be raised.
\end{abstract}

KEYWORDS: Papanicolaou test; Women's health; Neoplasm of cervix; Emotions.

\section{LA NO COMPARECENCIA AL EXAMEN PAPANICOLAO DEL CÁNCER DE CUELLO UTERINO: RAZONES DECLARADAS Y SENTIMIENTOS INVOLUCRADOS}

\begin{abstract}
RESUMEN: El cáncer del cuello uterino presenta altas tajas de prevalencia y mortalidad y la principal estrategia para su investigación es el examen citopatológico. Este estudio tuvo por objetivo conocer los motivos por los cuales ocurre la no comparecencia al examen en una Unidad de Salud de la Familia de Porto Alegre - Rio Grande do Sul. Es un estudio cualitativo, cuyos datos fueron recogidos en abril de 2011 por medio de entrevista semiestructurada y cuestionario sociodemográfico, aplicados a ocho sujetos. Los datos fueron analizados por la técnica de análisis de contenido, revelando multiplicidad de razones y sentimientos involucrados en la realización del examen citopatológico, principalmente del campo de la sexualidad y de la finitud de la vida. Se considera indispensable el vínculo entre profesionales y usuarias para que los sentimientos puedan ser minimizados y haya mayor adesión al proceso preventivo de esa enfermedad.

PALABRAS-CLAVE: Muestra vaginal; Salud de la mujer; Neoplasias del cuello del útero; Emociones.
\end{abstract}

\footnotetext{
*Trabalho de conclusão de curso apresentado ao Programa de Residência Multiprofissional em Saúde da Família e Comunidade, da Pontifícia Universidade Católica do Rio Grande do Sul - PREMUS09/PUCRS.

${ }^{1}$ Enfermeira. Especialista em Saúde da Família e Comunidade.

${ }^{2}$ Psicóloga. Psicanalista em formação. Mestre em Psicologia Clínica. Professora da Faculdade de Psicologia da PUCRS. Tutora do PREMUS09/PUCRS.

${ }^{3}$ Enfermeira. Mestre em Enfermagem. Professora da Faculdade de Enfermagem da PUCRS. Coordenadora/tutora do PREMUS09/ PUCRS.
}

Autor correspondente:

Recebido: 21/09/2011

Marcele Peretto

Aprovado: 24/01/2012

Pontifícia Universidade Católica do Rio Grande do Sul

Rua Sapé, 316 - 91350-050 - Porto Alegre-RS-Brasil

E-mail: enfermeiramar@gmail.com 


\section{INTRODUÇÃO}

O câncer do colo de útero é uma das neoplasias que apresenta um dos mais altos potenciais de cura, quando diagnosticado e tratado precocemente ${ }^{(1)}$. Contudo, apresenta altas taxas de prevalência e mortalidade, configurando-se em um grave problema de saúde pública, principalmente nos países em desenvolvimento ${ }^{(2)}$.

A Organização Mundial da Saúde estima a ocorrência de 500 mil novos casos de Câncer do Colo do Útero (CCU) por ano no mundo ${ }^{(1)}$. No Brasil, mesmo que nas últimas décadas tenha se buscado a reorganização da atenção básica - por meio da Estratégia de Saúde da Família e do aprimoramento do atendimento à saúde da mulher - o número de casos da doença ainda é preocupante ${ }^{(3)}$. O Instituto Nacional de Câncer estima que essa seja a terceira neoplasia mais comum entre as mulheres, superada apenas pela de pele (não-melanoma) e de mama, sendo a quarta causa de morte por câncer nessa população. No ano de 2010, estimativas apontaram a ocorrência de 18.430 mil novos casos, com um risco aproximado de 18 casos a cada 100 mil mulheres no país ${ }^{(1)}$.

Na Região Sul, sem considerar os tumores de pele não-melanoma, o CCU ocupa a terceira posição de incidência (21/100.000), especificamente no Rio Grande do Sul, estima-se que tenham ocorrido 1.250 novos casos, dos quais 210 somente na cidade de Porto Alegre $^{(1)}$. Ainda, no ano de 2008, foram internadas 409 mulheres com CCU nesse município, sendo 56 o número de óbitos associados ${ }^{(4)}$.

Quanto à etiologia, as evidências indicam que alguns tipos do Vírus do Papiloma Humano (HPV), transmitido sexualmente, são os principais agentes causadores do $\mathrm{CCU}^{(5)}$. Além disso, outros fatores contribuem para o aparecimento da doença: tabagismo, baixa ingestão de vitaminas, uso de contraceptivos orais, iniciação sexual precoce, multiplicidade de parceiros sexuais, multiparidade e coinfecção por agentes como o Vírus da Imunodeficiência Humana (HIV) e a Chlamydia trachomatis ${ }^{(1)}$.

Na fase pré-clínica do CCU tem-se a possibilidade de detectar lesões precursoras por meio da realização do exame citopatológico (CP), principal estratégia de rastreamento preconizada pelo Ministério da Saúde no Brasil ${ }^{(1,6)}$. Recomenda-se que esse exame seja feito anualmente em mulheres de 25 a 60 anos de idade e, após dois exames consecutivos com diagnóstico dentro da normalidade, seja realizado novo exame a cada três anos. Essa recomendação baseia-se na observação da história natural da neoplasia, que apresenta lenta progressão para a doença mais grave $\mathrm{e}^{(7)}$.

Considera-se que a prioridade etária para a detecção precoce é de 35 a 49 anos, pois esse é o período correspondente ao pico de incidência das lesões precursoras, o qual antecede ao pico de mortalidade pela doença ${ }^{(8)}$. Contudo, não estão sendo alcançadas as mulheres na faixa etária de maior risco, pois a maioria das que realizam o CP tem menos de 35 anos - provavelmente devido ao comparecimento aos serviços de saúde para cuidados relativos à natalidade, ocorrendo um subaproveitamento da rede de atenção ${ }^{(2)}$.

Além disso, embora esse método de rastreamento seja seguro, sensível e disponível nas Unidades Básicas de Saúde, estima-se que cerca de $40 \%$ das mulheres brasileiras nunca o tenham feito ${ }^{(9)}$. O Ministério da Saúde identificou que o desconhecimento da mulher sobre o câncer, o baixo nível de escolaridade, a falta de conhecimento sobre o próprio corpo, a vergonha e o medo de fazer o exame são algumas das barreiras para realizá-1o ${ }^{(8)}$.

Diante do exposto, o estudo objetivou conhecer os motivos da não realização da coleta do $\mathrm{CP}$ por mulheres de uma Unidade de Saúde da Família do Município de Porto Alegre/RS. Como objetivos específicos apresentam-se os de conhecer o significado emocional atribuído pelas usuárias frente à realização do exame, averiguar o conhecimento delas sobre a importância de realizá-lo, e verificar as informações que possuem acerca do CCU.

\section{MÉTODO}

Trata-se de um estudo de abordagem qualitativa, do tipo exploratório, utilizando-se como técnicas de coleta de dados a entrevista semiestruturada e o questionário sociodemográfico. Para analisar e interpretar os dados utilizou-se o método da análise de conteúdo, sendo o número de entrevistas delimitado por saturação de dados. Foi desenvolvido em uma Unidade de Saúde da Família, situada no Distrito Nordeste do Município de Porto Alegre/RS, contando com a participação de oito mulheres, de idade entre 30 e 56 anos, que não realizavam o exame citopatológico há pelo menos três anos.

Para a escolha das participantes, fez-se uma lista com nome e endereço de todas as mulheres que marcaram CP na Unidade nos meses de janeiro a dezembro de 2010. Em seguida, fez-se uma checagem no livro de registros de exames, produzindo-se uma nova lista 
com os dados daquelas que não haviam comparecido. Posteriormente, os prontuários das usuárias faltantes foram conferidos, a fim de identificar se preenchiam todos os critérios de inclusão para o estudo.

Deste modo, foram selecionadas as mulheres que ainda residiam na área de abrangência da Unidade; estavam na faixa etária preconizada para a realização do exame; já haviam mantido relações sexuais; já tinham feito o $\mathrm{CP}$ pelo menos uma vez; não realizavam o exame há pelo menos três anos, e que aceitaram e formalizaram a participação na pesquisa.

A coleta de dados foi realizada durante o mês de abril de 2011. Para a análise do conteúdo, seguiramse as etapas: reparação das informações - leitura de todos os materiais transcritos e decisão sobre quais estão de acordo com os objetivos da pesquisa; definição das unidades de análise; categorização, descrição e interpretação dos dados ${ }^{(10)}$. A partir das referidas etapas obtiveram-se quatro categorias: "Obstáculos na realização do exame citopatológico: razões declaradas e sentimentos envolvidos"; "Exame citopatológico: "porque tem que se prevenir"; "Câncer do colo do útero e outras doenças"; e "Relação profissional e usuária: o vínculo faz a diferença?".

Quanto aos aspectos éticos, o projeto foi submetido aos Comitês de Ética e Pesquisa da Secretaria Municipal de Saúde de Porto Alegre e da Pontifícia Universidade Católica do Rio Grande do Sul, tendo sido aprovado por ambos sob os protocolos n.001.004476.11.5 e 11/05378, respectivamente.

O estudo atendeu à Resolução 196/96 do Conselho Nacional de Saúde, tendo sido aplicado a cada entrevistada o Termo de Consentimento Livre e Esclarecido. Para preservar o anonimato das participantes, utilizou-se o sistema alfanumérico para a identificação dos relatos.

\section{RESULTADOS}

\section{Caracterização das participantes}

Das oito participantes do estudo, a maioria tinha um relacionamento estável e morava com o companheiro há pelo menos três anos, com exceção de uma, que estava separada. A idade de início da atividade sexual variou entre 11 e 23 anos de idade. Todas participantes tinham filhos, no mínimo um e no máximo cinco. O nível de escolaridade variou desde o ensino fundamental incompleto (quatro participantes) até o ensino médio completo (uma participante), três trabalhavam fora do ambiente doméstico e as demais no lar.

\section{Obstáculos na realização do exame citopatológico: razões declaradas e sentimentos envolvidos}

A realização do CP é uma ferramenta relevante para a detecção do CCU, tendo em vista seu importante papel para o diagnóstico precoce dessa doença. Porém, embora esse exame seja ofertado nos serviços públicos de saúde, há um grande número de mulheres que não o realizam periodicamente, tampouco o tenham feito alguma vez.

Nesse contexto, a atual pesquisa identificou inicialmente as razões declaradas pelas participantes e os sentimentos envolvidos nesse processo, para melhor compreendê-lo. Para tanto, questionou-se quais foram os motivos que as impediram de realizar o CP nos últimos anos. O descuido consigo mesma e a dificuldade para marcar a consulta, devido ao horário de trabalho, foram relatados como os principais obstáculos:

[...] eu acredito um pouco que a gente relaxa, entendeu? Porque, quando a gente quer fazer mesmo, a gente acha um jeito e vai fazer. (E1)

[...] eu preciso consultar, só que não tem horário pra mim aqui. Tenho que trabalhar! (E5)

Outro motivo apontado pelas entrevistadas foi o não reconhecimento de si mesma da necessidade de fazer o CP, expresso nas falas a seguir:

Porque eu achei que não era necessário [...] não vi nada assim de anormal prá ir fazer. (E6)

[...] não sinto nada, né? Daí, a gente sempre vai deixando. (E7)

Quando perguntadas de como se sentiam antes de realizar o CP, todas expressaram ansiedade relativa ao exame, sensação traduzida nos depoimentos pelo relato de nervosismo, tensão, relacionados tanto ao procedimento em si quanto ao possível resultado do exame:

[...] até subir na mesa, eu fico muito tensa. Eu não sei o porquê, mas eu fico. (E3)

Eu me sinto nervosa, né? Agora faz tempo que eu não faço, a gente fica pensando 'ah, será que tá tudo bem?' (E1) 
Ao serem questionadas acerca de como se sentiam no momento do exame, houve uma multiplicidade de sentimentos despertados. Algumas entrevistadas referiram se sentirem envergonhadas e desconfortáveis, enquanto outras relataram sentir dor.

[...] eu tenho um pouco de vergonha também. Vergonha! É desconfortável. (E5)

Eu não gosto de fazer esse exame, eu acho ele muito chato! É como se alguém tivesse fazendo uma coisa bem inconveniente, sabe? (E4)

Com a pessoa mais atenciosa, mas eu sinto dor. (E7)

A palavra medo foi a mais citada pelas entrevistadas, tanto no que se refere ao momento do exame quanto à expectativa do resultado:

Eu fiquei com medo, medo da coleta. (E6)

Tenho medo que apareça alguma coisa além, um cheiro esquisito, sabe? Coisas assim, coisas de mulher! (E8)

Do exame mesmo eu não tenho medo, sempre fui bem tratada, normal. É só medo do resultado mesmo. (E7)

Observa-se, ainda, que o medo não é referente apenas ao diagnóstico do CCU, visto que a maioria das participantes o associou a outras doenças, principalmente àquelas transmitidas sexualmente:

Vou sentir um medinho de fazer [...] dela falar 'ó, tu tá com uma infecção'. (E1)

Eu tenho vontade de fazer logo, saber logo o resultado [...]. Quem tem uma vida ativa [sexualmente] tem medo, né? (E7)

As participantes também expuseram suas percepções acerca de como gostariam que fosse o exame. Referiram a preferência pelo profissional do sexo feminino, além de mencionarem que gostariam que fosse feito em outro lugar do corpo, ou que não precisassem fazê-lo. Ainda, outras disseram que queriam que tivesse um meio mais fácil de diagnosticar o CCU, conforme os depoimentos que seguem:

Eu queria que fosse feito em outro lugar. (E2)
Alguém vai construir um aparelho, igual a um vaso sanitário, onde a mulher senta ali e saia toda a parte interna fotografada [...] prá não precisar fazer [0 CP]. (E4)

[...] prá mim, tendo uma mulher ali, eu já me sinto mais tranquila. (E8)

\section{Exame citopatológico: "porque tem que se prevenir"}

Quando interrogadas a respeito da finalidade do exame, seis participantes falaram sobre prevenção, embora citassem predominantemente que o $\mathrm{CP}$ seria feito para diagnosticar outras doenças e outros tipos de câncer. Também referiram não saber quais doenças poderiam ser identificadas por meio desse exame. Apenas uma entrevistada citou que seria para prevenir especificamente o CCU.

Eu sei que se faz prá saber se tem alguma doença, né? Agora, qual doença seria, eu não tenho ideia. (E1)

Prevenir se tiver alguma doença [...] o câncer em si. (E7)

Além disso, associaram a realização do exame ao cuidado com uma parte do corpo feminino. Contudo, as participantes não conseguiram dar nome a essa parte, apontando a genitália feminina.

[...] eu já ouvi falar que é o cuidado da parte do corpo da mulher, né? (E3)

\section{Câncer do colo do útero e outras doenças}

Quanto ao conhecimento que as entrevistadas possuem acerca do $\mathrm{CCU}$, constatou-se que a maioria associa essa doença ao sofrimento, mutilação e morte:

[...] uma experiência horrorosa, muito cruel [...]. Muitas vezes, perder o útero. (E4)

A única coisa que eu sei é que dá e mata a pessoa. (E6)

Por outro lado, surgiram falas a respeito do tratamento e da cura, embora essas remetam a um pensamento fantasioso por parte da entrevistada:

Se tratou [...] fez a quimioterapia, depois fez a rádio. 
Mas agora, tá lá, linda e formosa [...]. Ficou até mais nova! (E5)

Algumas participantes também revelaram raramente ouvir informações a respeito da doença, além de relatarem saber de outros cânceres, mas não especificamente sobre o do colo uterino.

É raro ouvir sobre isso [...]. Ouço mais sobre o da mama. (E2)

Só de ouvir a palavra câncer eu já fico aterrorizada, não importa onde. Mas especialmente sobre o colo do útero, eu não sei. (E7)

\section{Relação profissional e usuária: o vínculo faz a di-} ferença?

Durante as entrevistas surgiram falas acerca da relação entre o profissional que realiza o $\mathrm{CP}$ e a usuária do serviço de saúde. As entrevistadas referiram sentirem-se mais seguras ao realizar o exame com um profissional já conhecido por elas, conforme as seguintes falas:

Quando tu pega uma médica que tu conhece, não precisa te preocupar. (E1)

[...] porque ela [a médica] é muito antiga aqui, parece que eu tenho mais segurança. A gente não vai tá mostrando [a genitália] prá qualquer uma enfermeira, né? (E5)

A postura acolhedora por parte do profissional que realiza o exame - caracterizando o vínculo - e o domínio da técnica adequada para realizá-lo também foram aspectos que surgiram nas entrevistas, expressos na fala que segue:

A enfermeira que fez a coleta me deixou bem tranquila. É que depende também da profissional que tá fazendo [...] tem que ser bem treinada, porque conforme a conversa dela tu te sente bem prá fazer o exame. (E6)

\section{DISCUSSÃO}

Observou-se que são diversos os motivos que levaram ou contribuíram para as entrevistadas não realizar o CP. Nesse contexto, perceberam-se os distintos sentimentos despertados em uma mesma mulher e, ao mesmo tempo, várias mulheres relataram sensações semelhantes, evidenciando a complexidade deste fenômeno.

Assim como em estudo realizado na região norte do Brasil, o descuido com a própria saúde foi um dos principais motivos apontados pelas participantes para a não realização do exame ${ }^{(11)}$. Os resultados também corroboram estudo realizado em Belém/PA, que constatou que muitas mulheres só procuram assistência à saúde quando já estão doentes. Os autores sugerem que isso acontece devido ao maior enfoque dado ao tratamento e não à prevenção das doenças por influência do modelo biomédico ainda predominante em nosso país ${ }^{(5)}$. Ainda existe a ideia de ser desnecessário fazê-lo, ser embaraçoso e ter dificuldade em marcá-lo, sendo essas as razões relatadas em outra pesquisa ${ }^{(12)}$.

No que se refere ao conhecimento acerca do CP, nem todas as entrevistadas souberam relatar sua finalidade específica; contudo, tinham conhecimento de que se tratava de um exame para prevenir doenças, dentre elas o câncer. As participantes de outro estudo também demonstraram conhecer o exame de forma incompleta, e quando perguntadas sobre o seu real objetivo, surgiram dúvidas se o mesmo detectaria somente o CCU ou outras doenças ${ }^{(13)}$.

As entrevistadas ainda associaram o $\mathrm{CP}$ à prevenção de doenças sexualmente transmissíveis e ao cuidado de uma parte do corpo da mulher; no entanto, nenhuma delas nomeou a genitália. Além disso, relataram que gostariam que o exame fosse feito em outro lugar do corpo. Sabe-se que, desde a Antiguidade, o mistério e o tabu cercam o corpo da mulher e que, mesmo na atualidade, a anatomia dos órgãos sexuais e reprodutivos femininos é desconhecida por muitas mulheres. Pondera-se que nomear o corpo feminino é também afirmar sua existência, sua complexidade, enquanto não reconhecer o próprio corpo pode implicar não reconhecer sua sexualidade, embora essa não se restrinja apenas aos órgãos sexuais ${ }^{(14)}$.

Assim, pensa-se na possibilidade de que algumas mulheres preferem não saber exatamente como é o exame - não se apropriando do mesmo - já que, neste momento, outra pessoa precisa observar uma região tão íntima, a qual, muitas vezes, ela própria desconhece e tem vergonha de olhar e tocar.

Os sentimentos de vergonha, nervosismo e medo também já foram relatados, esses sentimentos podem ser justificados pelo tabu do sexo, proveniente da educação recebida e da falta de informação ${ }^{(15)}$; medo, vergonha e dor na realização do $\mathrm{CP}$ parecem estar 
diretamente ligados com a questão do pudor ${ }^{(3)}$. Devido a isso, algumas das entrevistadas disseram sentir-se mais tranquilas na presença de um profissional do sexo feminino, provavelmente pela semelhança física, pela possibilidade de compartilhar dos mesmos sentimentos ante o exame, e da característica erótica que pode estar presente na relação homem/mulher. Um estudo constatou a mesma situação, no qual as mulheres relataram ser mais fácil enfrentar o exame quando o profissional é do mesmo sexo ${ }^{(16)}$.

Embora esses motivos e sentimentos relatados tenham sido múltiplos, parece que a maioria esbarra em obstáculos oriundos de um mesmo território: o da sexualidade. A maneira como algumas mulheres reagem ao ter que expor seu corpo, tê-lo manipulado e examinado por um profissional, revela a importância da sexualidade, pois se trata de tocar e manusear órgãos e zonas erógenas ${ }^{(15)}$, mesmo que não tenha nenhuma conotação sexual.

Ainda, compreende-se que um possível diagnóstico de uma doença que carrega tantos estigmas também contribua para a não realização de um exame que possa detectá-la. Sabe-se que o câncer está vinculado ao imaginário social como uma doença incurável, com a qual a mulher não teria chances de sobreviver ${ }^{(5)}$. Nesse sentido, a falta de um conhecimento adequado em relação ao CCU e ao real valor de detectá-lo precocemente desencoraja as mulheres a procurarem o exame, justamente por medo de descobrirem-se com a doença e terem de deparar-se com a finitude da vida. Destaca-se que, diante do diagnóstico de câncer, os sentimentos de desesperança, angústia e medo são comuns, revelando a vulnerabilidade emocional da mulher frente à possibilidade da morte ${ }^{(17)}$.

Em relação ao vínculo profissional-usuária, considera-se relevante a interação mútua entre esse binômio, para que as usuárias possam falar de si, de seus sentimentos, de suas vivências e experiências ${ }^{(3)}$. Entende-se, também, que é necessário que o profissional desenvolva um clima de empatia, agindo não somente com o preparo técnico, mas com intuição e sensibilidade, buscando estabelecer um vínculo de confiança ${ }^{(13,18)}$.

Seguramente, o estabelecimento deste vínculo propiciaria um maior diálogo com as usuárias, permitindo a abordagem de questões relativas ao conhecimento do próprio corpo, de sua anatomia, seu funcionamento e de suas representações psicológicas, contribuindo para desmistificar e quebrar os tabus que envolvem o corpo da mulher e sua relação com o sexo ${ }^{(14)}$, já que parece ser essa uma das principais barreiras para a não realização do $\mathrm{CP}$.

Ainda, ressalta-se a importância desse profissional enquanto educador, incentivando-as na prática do exame e fortalecendo sua participação social no processo, conscientizando-as de seu papel de sujeitos responsáveis por sua saúde e bem-estar ${ }^{(19)}$. Pensa-se que, dessa maneira, seja possível minimizar a dor, a vergonha, a ansiedade, o medo e os demais sentimentos já relatados frente à realização do $\mathrm{CP}^{(3,18)}$.

Neste contexto, considera-se, também, que toda a equipe de saúde tem a contribuir na melhora da adesão ao processo preventivo do CCU tanto no acolhimento e na escuta quando a usuária procura o serviço, quanto na realização do exame e na orientação acerca da real importância de fazê-lo e de seu impacto na saúde mulher. Dessa forma, pensa-se no trabalho interdisciplinar como estratégia para a prevenção da doença e para a promoção da saúde.

A Enfermagem, especificamente, tem muito a contribuir nesse sentido, pois tem sua formação pautada na educação para a saúde e no trabalho integrado com outras profissões. Ainda, por ser uma profissão predominantemente feminina, pode ter maior proximidade com esse complexo universo de sentimentos envolvidos na realização do $\mathrm{CP}$ e, dessa forma, ter a oportunidade de sensibilizar mais usuárias na adesão ao processo de prevenção ao CCU.

\section{CONSIDERAÇÕES FINAIS}

A presente pesquisa propôs-se a avaliar a complexidade do fenômeno que envolve a não realização do exame citopatológico. Sabe-se que muitas questões estão entrelaçadas nesse contexto. Portanto, questiona-se: estaria o tabu da sexualidade reforçando o desconhecimento do próprio corpo e levando muitas mulheres a não realizar o exame? O medo e a angústia sentidos frente à possibilidade do diagnóstico positivo para $\mathrm{o}$ CCU estariam relacionados à vulnerabilidade humana diante da finitude da vida?

Considera-se que para amenizar os múltiplos sentimentos envolvidos seja imprescindível o vínculo entre profissionais e usuárias dos serviços de saúde. Salienta-se, deste modo, a relevância da temática para a Enfermagem, já que na rede pública de saúde o $\mathrm{CP}$ é, na maioria das vezes, realizado pelo enfermeiro. Ainda, acredita-se que a realização de um trabalho interdisciplinar seja um facilitador com vistas à diminuição dos tabus da sexualidade e ao estímulo ao 
conhecimento do próprio corpo, de seu funcionamento e de suas representações emocionais para que, dessa forma, possa-se realmente impactar na prevenção do CCU e na promoção da saúde dessas mulheres.

Por fim, considera-se que não basta disponibilizar o exame CP nos serviços de saúde, tampouco lançar informações acerca do mesmo. Antes, é necessário garantir que a mulher tenha acesso a essas informações, e que as compreenda - seja, por exemplo, com a busca ativa das faltosas ou com campanhas de incentivo à realização do $\mathrm{CP}$. Deste modo, acredita-se que haverá maiores chances de que ela (re)pense seus saberes e conscientize-se da verdadeira importância do exame e, assim, busque efetivamente realizá-lo.

\section{REFERÊNCIAS}

1. Ministério da Saúde(BR). Instituto Nacional do Câncer. Estimativa 2010: incidência de câncer no Brasil. Rio de Janeiro: INCA; 2009.98 p.

2. Albuquerque KM, Frias PG, Andrade CLT, Aquino EML, Menezes G, Szwarcwald CL. Cobertura do teste de Papanicolaou e fatores associados à não-realização: um olhar sobre o programa de prevenção do câncer do colo do útero em Pernambuco, Brasil. Cad. saude publica. 2009;25(Suppl 2):S301-9.

3. Brito CMS, Nery IS, Torres LC. Sentimentos e expectativas das mulheres acerca da Citologia Oncótica. Rev. bras. enferm. 2007;60(4):387-90.

4. Prefeitura Municipal de Porto Alegre. Equipe de Vigilância de Eventos Vitais, Doenças e Agravos não Transmissíveis. Sistema de Informação do Câncer do Colo do Útero - SISCOLO. Relatório 2008. Porto Alegre; 2008. 09 p.

5. Silva SED, Vasconcelos EV, Santana ME, Rodrigues ILA, Mar DF, Carvalho FL. Esse tal Nicolau: representações sociais de mulheres sobre o exame preventivo do câncer cérvico-uterino. Rev. esc. enferm. USP. 2010;44(3):554-60.

6. Vasconcelos CTM, Neto JAV, Castelo ARP, Medeiros FC, Pinheiro AKB. Análise da cobertura e dos exames colpocitológicos não retirados de uma Unidade Básica de Saúde. Rev. esc. enferm. USP. 2010;44(2):324-30.

7. Ministério da Saúde (BR). Secretaria de Atenção à Saúde. Departamento de Atenção Básica. Controle dos cânceres do colo do útero e da mama. Brasília: Ministério da Saúde; 2006. 131 p.

8. Ministério da Saúde (BR). Secretaria Nacional de Assistência à Saúde. Instituto Nacional do Câncer INCA. Falando sobre câncer do colo do útero. Rio de Janeiro: INCA; 2002. 56 p.

9. Cruz LMB, Loureiro RP. A comunicação na abordagem preventiva do câncer do colo do útero: importância das influências histórico-culturais e da sexualidade feminina na adesão às campanhas. Saude soc. 2008;17(2):120-31.

10. Moraes R. Análise de conteúdo. Rev. educ. 1999;22(37)7-32.

11. Fernandes JV, Rodrigues SHL, Costa YGAS, Silva LCM, Brito AML, Azevedo JWV, et al. Conhecimentos, atitudes e prática do exame de Papanicolau por mulheres, Nordeste do Brasil. Rev. saude publica. 2009;43(5):851-8.

12. Amorim VMSL, Barros MBA, César CLG, Carandina L, Goldbaum M. Fatores associados a não realização do exame de Papanicolaou: um estudo de base populacional no Município de Campinas, São Paulo, Brasil. Cad. saude publica. 2006;9(3):325-34.

13. Oliveira SL, Almeida ACH. A percepção das mulheres frente ao exame de Papanicolau: da observação ao entendimento. Cogitare enferm. 2009;14(3):518-26.

14. Melo MCB, Barros EN. Histerectomia e simbolismo do útero: possíveis repercussões na sexualidade feminina. Rev. SBPH. 2009;12(2):80-99.

15. Duavy LM, Batista FLR, Jorge MSB, Santos JBF. A percepção da mulher sobre o exame preventivo do câncer cérvico-uterino: estudo de caso. Cienc. saude colet. 2007;12(3):733-42.

16. Ferreira MLSM. Motivos que influenciam a nãorealização do exame de papanicolaou segundo a percepção de mulheres. Esc. Anna Nery. 2009;13(2):37884.

17. Araújo IMA, Fernandes AFC. O significado do diagnóstico do câncer de mama para a mulher. Esc. Anna Nery. 2008;12(4):664-71.

18. Merighi MAB, Hamano L, Cavalcante LG. O exame preventivo do câncer cérvico-uterino: conhecimento e significado para as funcionárias de uma escola de enfermagem de uma instituição pública. Rev. esc. enferm. USP. 2002;36(3):289-96.' 
19. Soares MC, Meincke SMK, Mishima SM, Simino GPR. Câncer de colo uterino: caracterização das mulheres em um município do Sul do Brasil. Esc. Anna Nery. 2010;14(1):90-6. 